\title{
Les résultats équivoques de la détection par PCR de Bordetella pertussis chez les enfants devraient-ils être signalés aux autorités de la santé publique?
}

\author{
M Desjardins 1,3 , S Mousseau², P Doyon-Plourde ${ }^{3,4}$, N Brousseau ${ }^{5}$, D lachimov ${ }^{4}$, F Rallu ${ }^{3,6}$, \\ C Quach $3,4,6,7 *$
}

\section{Résumé}

Introduction : L'amplification en chaîne par polymérase (polymerase chain reaction [PCR]) en temps réel est la méthode privilégiée pour la détection de la coqueluche. Au Québec, les résultats positifs et équivoques doivent être signalés aux autorités de la santé publique; par contre, ce n'est pas le cas en Ontario.

Objectif : Établir l'importance clinique des résultats équivoques comparativement aux résultats positifs chez les enfants avec suspicion clinique de coqueluche.

Méthodologie : Étude de cohorte rétrospective de patients consécutifs vus au Centre hospitalier universitaire Sainte-Justine à Montréal (Québec) chez qui une coqueluche était suspectée et ayant fait l'objet d'analyse par PCR multiplex bactérienne (notamment pour Bordetella pertussis) entre 2015 et 2017. Les dossiers médicaux ont été examinés à l'aide d'un formulaire normalisé. Des analyses univariées (test T de Student et test du chi carré) et des analyses multivariées par régression logistique ont été utilisées pour comparer les cas de résultats positifs et équivoques.

Résultats : Des 1526 analyses par PCR multiplex réalisées, 109 étaient positives et 24 étaient équivoques. Les deux groupes étaient semblables quant aux données démographiques et aux évaluations de la gravité de la maladie, mais les patients du groupe dont les résultats étaient équivoques étaient moins nombreux à présenter une toux paroxystique $(33,3 \% \mathrm{c}$. $79,8 \%$; rapport de cotes $[R C]$ ajusté : 0,11 ; intervalle de confiance [IC] à $95 \%: 0,04-0,29)$ et un chant du coq (0 \% c. 18,3\%; $p<0,001)$, leur numération lymphocytaire était inférieure $(6,6$ c. $\left.11,9 \times 10^{\%} / \mathrm{L} ; p=0,008\right)$, ils étaient plus susceptibles de recevoir un diagnostic de co-infection virale $(16,7 \%$ c. $3,7 \%$; RC ajusté : 5,62; IC à $95 \%: 1,17-27,54)$ et moins susceptibles de recevoir un macrolide (25\% c. $89 \%$; RC ajusté : 0,04; IC à $95 \%$ : 0,01-0,11). La durée moyenne du séjour des patients admis ayant obtenu des résultats équivoques était plus courte $(3,3 \mathrm{c}$. 12,2 jours; $p=0,001$ ).

Conclusion : Malgré des similitudes quant à la gravité de la maladie, les manifestations cliniques chez les enfants avec suspicion clinique de coqueluche ayant obtenu des résultats équivoques à la PCR était significativement différent de celui observé chez les enfants ayant obtenu des résultats positifs. Dans un contexte où les ressources de santé publique sont limitées, ce constat peut orienter la décision concernant la nécessité de signaler ou non les résultats équivoques aux autorités de la santé publique.

\section{Affiliations}

${ }^{1}$ Microbiologie médicale et infectiologie, Centre hospitalier de I'Université de Montréal, Montréal (Québec)

2 Service d'urgence pédiatrique, Département de pédiatrie, Centre hospitalier universitaire Sainte-Justine, Montréal (Québec)

${ }^{3}$ Département de microbiologie, infectiologie et immunologie, Faculté de médecine, Université de Montréal, Montréal (Québec)

${ }^{4}$ Centre de recherche, Centre hospitalier universitaire Sainte-Justine, Montréal (Québec)

${ }^{5}$ Direction des risques biologiques et de la santé au travail, Institut national de santé publique du Québec, Québec (Québec)

${ }^{6}$ Département de microbiologie médicale, Centre hospitalier universitaire Sainte-Justine, Montréal (Québec)

${ }^{7}$ Prévention et contrôle des infections, Centre hospitalier universitaire Sainte-Justine, Montréal (Québec)

*Correspondance : c.quach@ umontreal.ca
Citation proposée : Desjardins M, Mousseau S, Doyon-Plourde P, Brousseau N, lachimov D, Rallu F, Quach C. Les résultats équivoques de la détection par PCR de Bordetella pertussis chez les enfants devraient-ils être signalés aux autorités de la santé publique? Relevé des maladies transmissibles au Canada 2018;44(9):221-6. https://doi.org/10.14745/ccdr.v44i09a02f

Mots-clés : coqueluche, pédiatrie, dépistage, résultats équivoques 


\section{Introduction}

La coqueluche est une maladie très contagieuse causée par Bordetella pertussis. Malgré la vaccination universelle, la coqueluche représente toujours un fardeau important pour la santé publique au Canada, en particulier chez les enfants de moins de 15 ans (1). Certains groupes, comme les nourrissons, présentent un risque accru d'atteinte grave. Par conséquent, les cas de coqueluche sont signalés aux autorités de la santé publique dans les 48 heures suivant le diagnostic aux fins de surveillance épidémiologique et de prise en charge des contacts (2).

Le diagnostic clinique de la coqueluche représente un défi en raison du large éventail de symptômes observés (3). La culture nasopharyngienne et l'amplification en chaîne par polymérase (polymerase chain reaction [PCR]) constituent deux méthodes de confirmation en laboratoire acceptées (4); cependant, compte tenu de sa sensibilité accrue, la PCR est devenue la méthode diagnostique privilégiée pour la coqueluche dans la plupart des provinces et territoires $(2,5)$.

Le Centre hospitalier universitaire Sainte-Justine (CHUSJ), un hôpital pédiatrique de soins tertiaires de Montréal, utilise la PCR en temps réel basée sur I'IS481 pour le diagnostic de $B$. pertussis. La PCR est considérée comme positive lorsque la valeur du cycle seuil (cycle threshold [Ct]) est inférieure à 36 et équivoque quand elle est de 36 à 39,9. Actuellement, au Québec, les laboratoires signalent les résultats équivoques aux autorités de la santé publique. Ces cas font l'objet d'investigations, notamment la recherche des contacts, et sont interprétés en fonction des caractéristiques cliniques (symptômes correspondant à la coqueluche) et des données épidémiologiques (antécédents de contact) (2). Par contre, en raison des questions soulevées sur la portée des résultats équivoques (5), Santé publique Ontario a décidé que ces résultats n'avaient pas à être déclarés (6). Notre étude visait à établir la portée clinique d'un résultat équivoque à la PCR, comparativement à un résultat positif, chez les enfants avec suspicion clinique de coqueluche qui ont fait l'objet d'une évaluation.

\section{Méthodologie}

\section{Conception de l'étude}

Il s'agit d'une étude d'observation rétrospective de cohorte menée auprès de patients consécutifs vus au CHUSJ en raison d'une coqueluche présumée et qui ont fait l'objet d'une PCR multiplex bactérienne (B. pertussis, B. parapertussis, B. holmesii, Mycoplasma pneumoniae et Chlamydophila pneumoniae) entre juin 2015 et mars 2017. Le protocole de l'étude a été approuvé par le comité d'éthique du CHUSJ.

\section{Participants de l'étude}

L'ensemble de la cohorte était composé de participants de 17 ans ou moins dont un résultat à la PCR multiplex bactérienne figurait dans le système d'information de laboratoire de microbiologie. Les patients ayant obtenu un résultat positif ou équivoque à la PCR pour $B$. pertussis ont été inclus dans notre cohorte, sans égard à l'endroit où l'analyse avait été commandée (urgence, service d'hospitalisation, clinique, unité de soins intensifs pédiatriques ou unité néonatale de soins intensifs). Les patients qui n'ont pas été évalués par un médecin du CHUSJ le jour où l'analyse a été effectuée ont été exclus, puisque les données concernant les manifestations cliniques (symptômes et signes) n'étaient pas disponibles.

\section{Collecte de données}

Deux membres de l'équipe de recherche (MD, DI) ont effectué un examen manuel des dossiers médicaux électroniques à l'aide de Chartmaxx (Quest Diagnostics, Secaucus, New Jersey, États-Unis [É.-U.]) pour tous les patients inclus dans la cohorte. Les données ont été extraites à l'aide d'un formulaire d'exposé de cas normalisé. Les deux chercheurs ont examiné dix pour cent des dossiers afin d'évaluer la concordance interévaluateurs à l'aide du test de concordance kappa. Les données recueillies comprenaient les caractéristiques démographiques, les antécédents médicaux, l'état vaccinal, les manifestations cliniques ainsi que la gravité et les issues de la maladie. Lorsqu'ils n'étaient pas consignés dans le dossier, les signes et symptômes spécifiques ainsi que les antécédents médicaux étaient considérés comme absents. La gravité de la maladie était établie à l'aide de deux scores de gravité : I'échelle de Preziosi modifiée (Modified Preziosi Scale [MPS]) et I'indice de gravité respiratoire (Respiratory Severity Score [RSS]). La MPS a été utilisée pour mesurer la gravité de la coqueluche chez les patients pédiatriques. Une maladie grave est définie par un résultat à l'échelle MPS supérieur à six (7). L'indice RSS permet d'évaluer la gravité des infections respiratoires chez les patients pédiatriques. II a été utilisé pour distinguer les infections des voies respiratoires supérieures et inférieures et corrèle avec la nécessité d'hospitaliser le patient (8). Comme ces deux scores mesurent différentes aspects des infections respiratoires, nous avons comparé les deux groupes de patients à l'aide des deux scores.

\section{Analyse des données}

Les analyses univariées, utilisant le test du chi carré et le test $T$ de Student selon le cas, ont d'abord été réalisées pour comparer les caractéristiques des patients ayant obtenu un résultat équivoque (par rapport à ceux ayant obtenu un résultat positif). Les variables statistiquement significatives dans l'analyse univariée, celles qui étaient considérées comme d'éventuelles variables de confusion ou qui avaient une incidence sur le critère d'information d'Akaike adapté au modèle, ont été incluses dans le modèle de régression logistique multivariable (rapports de cotes [RC] et intervalles de confiance [IC] à $95 \%$ ). Toutes les valeurs $p$ étaient bilatérales et considérées comme significatives à moins de 0,05 (logiciel SPSS, version 24, IBM Analytics, Armonk, New York, É-U et R, version 3.4.3).

\section{Résultats}

Au total, 1526 analyses par PCR multiplex bactériennes consécutives dont les résultats étaient disponibles, réalisées au CHUSJ entre le 11 juin 2015 et le 31 mars 2017, ont été extraites 
du système d'information de laboratoire. Parmi ces analyses, 109 patients ont obtenu un résultat positif pour $B$. pertussis et 24, un résultat équivoque. Les deux groupes étaient semblables quant aux données démographiques (âge, sexe) et aux antécédents médicaux (antécédents d'asthme, prématurité), à l'exception d'un taux de vaccination inférieur chez les patients ayant obtenu un résultat positif à la PCR (tableau 1). La proportion de patients ayant des antécédents de contact avec un cas de coqueluche était également similaire.

Tableau 1 : Caractéristiques démographiques et antécédents médicaux des patients

\begin{tabular}{|c|c|c|c|c|}
\hline Caractéristiques & $\begin{array}{c}\text { Résultat } \\
\text { positif à la } \\
\text { PCR } \\
n=109(\%)^{a}\end{array}$ & $\begin{array}{c}\text { Résultat } \\
\text { équivoque } \\
\text { à la PCR } \\
n=24(\%)^{a}\end{array}$ & $\begin{array}{l}\text { Valeur } \\
p\end{array}$ & $\begin{array}{c}\text { RC } \\
\text { (IC à } 95 \%)\end{array}$ \\
\hline Âge, moyen (É.-T.) & $6,65(5,35)$ & $6,26(5,23)$ & 0,75 & S.O. \\
\hline Sexe masculin & $45(41,3)$ & $11(45,8)$ & 0,68 & $1,20(0,49-2,93)$ \\
\hline Vaccination à jour & $79(72,5)$ & $22(91,7)$ & 0,046 & $4,18(1,13-27,0)^{b}$ \\
\hline Asthme & $16(14,7)$ & $7(29,2)$ & 0,09 & $2,39(0,82-6,56)$ \\
\hline Prématurité & $5(4,6)$ & $3(12,5)$ & 0,14 & $2,97(0,57-13,08)$ \\
\hline Immunodépression & $0(0,0)$ & $0(0,0)$ & S.O. & S.O. \\
\hline $\begin{array}{l}\text { Contact avec un } \\
\text { cas de coqueluche }\end{array}$ & $18(16,5)$ & $3(12,5)$ & 0,63 & $0,72(0,16-2,39)$ \\
\hline \multicolumn{5}{|c|}{ Emplacement du patient } \\
\hline Urgence & $89(81,7)$ & $16(66,7)$ & $0,10^{c}$ & $0,44(0,17-1,23)^{c}$ \\
\hline $\begin{array}{l}\text { Service } \\
\text { d'hospitalisation }\end{array}$ & $14(12,8)$ & $7(29,2)$ & - & - \\
\hline Clinique & $5(4,6)$ & $1(4,2)$ & - & - \\
\hline UNSI & $0(0,0)$ & $0(0,0)$ & - & - \\
\hline USIP & $1(0,9)$ & $0(0,0)$ & - & - \\
\hline \multicolumn{5}{|c|}{ Spécialité du médecin prescripteur } \\
\hline $\begin{array}{l}\text { Pédiatrie } \\
\text { d'urgence }\end{array}$ & $55(50,5)$ & $8(33,3)$ & $0,13^{d}$ & $0,49(0,19-1,21)^{d}$ \\
\hline Pédiatrie & $43(39,4)$ & $12(50,0)$ & - & - \\
\hline Infectiologie & $5(4,6)$ & $3(12,5)$ & - & - \\
\hline Médecine familiale & $6(5,5)$ & $0(0,0)$ & - & - \\
\hline Autre spécialité & $0(0,0)$ & $1(4,2)$ & - & - \\
\hline
\end{tabular}

Abréviations : É.-T., écart-type; IC, intervalle de confiance; PCR, polymerase chain reaction ou amplification en chaîne par polymérase; RC, rapport de cotes; S.O., sans objet; UNSI, unité néonatale de soins intensifs; USIP, unité de soins intensifs pédiatriques; « - », inclus dans le calcul au-dessus

À l'exception de l'âge, qui est indiqué sous forme de moyenne et d'écart-type

${ }^{b}$ Résultats statistiquement significatifs $(p<0,05)$

' Urgence par rapport aux autres lieux

¿ Pédiatrie d'urgence par rapport aux autres spécialités

Les signes et symptômes observés lors des investigations menées en laboratoire et en radiologie (paracliniques) sont présentés dans le tableau 2. Lors de la comparaison des deux groupes, aucune différence n'a été observée pour ce qui est de la fièvre, de la rhinorrhée, de la toux, de la toux émétisante, des anomalies à l'auscultation, de la respiration sifflante, de l'otite moyenne aiguë et de la pharyngite. Les scores à la MPS étaient également similaires dans les deux groupes. Toutefois, les patients du groupe ayant obtenu des résultats positifs à la PCR étaient significativement plus nombreux à présenter une toux paroxystique, un chant du coq et une lymphocytose et moins nombreux à présenter une rhinorrhée et un tirage que
Tableau 2 : Manifestations cliniques et investigations

\begin{tabular}{|c|c|c|c|c|c|}
\hline Présentation & $\begin{array}{c}\text { Résultats } \\
\text { positifs à la } \\
\text { PCR } \\
n=109(\%)^{a}\end{array}$ & $\begin{array}{l}\text { Résultats } \\
\text { équivoques } \\
\text { à la PCR } \\
n=24(\%)^{a}\end{array}$ & $\begin{array}{c}\text { Valeur } \\
p\end{array}$ & $\begin{array}{c}\text { RC brut } \\
\text { (IC à } \\
95 \%)\end{array}$ & $\begin{array}{c}\text { RC ajusté } \\
\text { (IC à } \\
95 \%)\end{array}$ \\
\hline \multicolumn{6}{|c|}{ Manifestations clinique } \\
\hline Fièvre & $10(9,2)$ & $5(20,8)$ & 0,10 & $\begin{array}{r}2,61 \\
(0,74-8,25)\end{array}$ & $\begin{array}{r}2,30 \\
(0,62-7,57)^{d}\end{array}$ \\
\hline Rhinorrhée & $31(28,4)$ & $12(50,0)$ & $0,04^{\mathrm{a}}$ & $\begin{array}{r}2,52 \\
(1,02-6,26)^{a}\end{array}$ & $\begin{array}{r}2,53 \\
(1,01-6,42)^{b, e}\end{array}$ \\
\hline $\begin{array}{l}\text { Apnée } \\
\text { confirmée }\end{array}$ & $10(9,2)$ & $0(0,0)$ & 0,12 & S.O. & S.O. \\
\hline $\begin{array}{l}\text { Durée de la } \\
\text { toux, jours } \\
(\text { É.-T.)c }\end{array}$ & $15,5(14,2)$ & $16,3(67,9)$ & 0,82 & S.O. & S.O. \\
\hline $\begin{array}{l}\text { Toux } \\
\text { émétisante }\end{array}$ & $55(50,5)$ & $8(33,3)$ & 0,13 & $\begin{array}{r}0,49 \\
(0,19-1,21)\end{array}$ & $\begin{array}{r}0,49 \\
(0,19-1,21)^{\mathrm{e}}\end{array}$ \\
\hline $\begin{array}{l}\text { Toux } \\
\text { paroxystique }\end{array}$ & $87(79,8)$ & $8(33,3)$ & $<0,001^{a}$ & $\begin{array}{r}0,13 \\
(0,05-0,32)^{\mathrm{b}}\end{array}$ & $\begin{array}{r}0,11 \\
(0,04-0,29)^{\mathrm{b}, e}\end{array}$ \\
\hline Chant du coq & $20(18,3)$ & $0(0,0)$ & $<0,001^{a}$ & S.O. & S.O. \\
\hline $\begin{array}{l}\text { Anomalies à } \\
\text { l'auscultation }\end{array}$ & $12(11,0)$ & $5(20,8)$ & 0,19 & $\begin{array}{r}2,13 \\
(0,62-6,49)\end{array}$ & $\begin{array}{r}2,18 \\
(0,60-7,19)^{\mathrm{e}}\end{array}$ \\
\hline $\begin{array}{l}\text { Respiration } \\
\text { sifflante }\end{array}$ & $2(1,8)$ & $2(8,3)$ & 0,09 & $\begin{array}{r}4,86 \\
(0,56-42,34)\end{array}$ & $\begin{array}{r}3,38 \\
(0,36-31,52)^{f}\end{array}$ \\
\hline $\begin{array}{l}\text { Tirage (tout } \\
\text { type) }\end{array}$ & $6(5,5)$ & $6(25,0)$ & $0,003^{a}$ & $\begin{array}{r}5,72 \\
(1,62-20,29)^{b}\end{array}$ & $\begin{array}{r}5,61 \\
(1,55-20,76)^{\mathrm{b}, 9}\end{array}$ \\
\hline $\begin{array}{l}\text { Otite moyenne } \\
\text { aiguë }\end{array}$ & $5(4,6)$ & $3(12,5)$ & 0,14 & $\begin{array}{r}2,97 \\
(0,57-13,08)\end{array}$ & $\begin{array}{r}1,72 \\
(0,26-8,92)^{\mathrm{h}}\end{array}$ \\
\hline Pharyngite & $9(8,3)$ & $1(4,2)$ & 0,49 & $\begin{array}{r}0,48 \\
(0,02-2,76)\end{array}$ & $\begin{array}{r}0,50 \\
(0,03-2,91)^{\mathrm{e}}\end{array}$ \\
\hline Cyanose & $15(13,8)$ & $4(16,7)$ & 0,71 & $\begin{array}{r}1,25 \\
(0,33-3,90) \\
\end{array}$ & $\begin{array}{r}0,69 \\
(0,13-3,16)^{f} \\
\end{array}$ \\
\hline \multicolumn{6}{|l|}{ Score de gravité } \\
\hline $\begin{array}{l}\text { Échelle MPS, } \\
\text { moyenne }\end{array}$ & 5,7 & 4,8 & 0,31 & S.O. & S.O. \\
\hline $\begin{array}{l}\text { Échelle MPS, } \\
\text { atteinte grave }\end{array}$ & $30(27,5)$ & $6(25,0)$ & 0,80 & $\begin{array}{r}0,88 \\
(0,30-2,32)\end{array}$ & $\begin{array}{r}0,40 \\
(0,06-1,86)^{i}\end{array}$ \\
\hline $\begin{array}{l}\text { Indice RSS, } \\
\text { moyenne }\end{array}$ & 0,5 & 1,3 & 0,07 & S.O. & S.O. \\
\hline \multicolumn{6}{|l|}{ Investigations } \\
\hline $\begin{array}{l}\text { Détection } \\
\text { d'agents } \\
\text { viraux par PCR } \\
\text { multiplex }\end{array}$ & $14(12,8)$ & $8(33,3)$ & 0,01 & $\begin{array}{r}3,39 \\
(1,19-9,33)\end{array}$ & $\begin{array}{r}3,03 \\
(0,82-11,35)^{f}\end{array}$ \\
\hline $\begin{array}{l}\text { Autre virus } \\
\text { détecté }\end{array}$ & $4(3,7)$ & $4(16,7)$ & 0,02 & $\begin{array}{r}5,25 \\
(1,16-23,92)^{\mathrm{b}} \\
\end{array}$ & $\begin{array}{r}5,62 \\
(1,17-27,54)^{b, e} \\
\end{array}$ \\
\hline $\begin{array}{l}\text { Lymphocytes } \\
\left.\text { (x } 10^{9} / \mathrm{L}\right), \\
\text { moyenne } \\
\text { (É.-T.) }\end{array}$ & $11,9(10,9)$ & $6,6(2,8)$ & $0,008^{b}$ & S.O. & S.O. \\
\hline $\begin{array}{l}\text { Pneumonie } \\
\text { visible à la } \\
\text { radiographie }\end{array}$ & $5(4,6)$ & $2(8,3)$ & 0,46 & $\begin{array}{r}1,89 \\
(0,26-9,41)\end{array}$ & $\begin{array}{r}3,34 \\
(0,41-22,40)^{j}\end{array}$ \\
\hline
\end{tabular}

Abréviations : É.-T., écart-type; IC , intervalle de confiance; MPS, Modified Preziosi Scale ou échelle de Preziosi modifiée; PCR, polymerase chain reaction ou amplification en chaîne par polymérase; RC, rapport de cotes; RSS, Respiratory Severity Score ou indice de gravité respiratoire; S.O., sans objet; < , inférieur à

a Tous les résultats sont indiqués sous forme de $\mathrm{n}(\%)$, à l'exception de la durée de la toux (jours) et du nombre de lymphocytes, qui sont indiqués sous forme de moyenne avec écart-type, et des scores de gravité à l'échelle MPS et à l'indice RSS, qui sont indiqués sous forme de moyenne ${ }^{b}$ Résultats statistiquement significatifs $(p<0,05)$

Trois données manquantes dans chaque groupe

Ajusté en fonction du groupe d'âge et de tout autre virus détecté

e Ajusté en fonction de l'âge

Ajusté en fonction de l'âge et de l'emplacement du patient

9justé en fonction de la vaccination à jour

h Ajusté en fonction de l'âge et de tout autre virus détecté

Ajusté en fonction de l'âge, de tout autre virus détecté et de l'emplacement du patient

Ajusté en fonction de l'âge et de la vaccination à jour 
les patients du groupe ayant obtenu des résultats équivoques à la PCR. De plus, aucune apnée n'a été signalée dans le dernier groupe. Enfin, les patients ayant obtenu des résultats équivoques à la PCR avaient tendance à faire plus souvent l'objet de tests de détection d'agents pathogènes viraux par PCR multiplex (RC ajusté : 3,03 $(0,82-11,35)$, avec une grande proportion d'infections virales confirmées (16,7 \% c. 3,7 \%; RC ajusté : 5,62; IC à $95 \%: 1,17-27,54)$.

Le tableau 3 présente les issues cliniques des patients. II convient de noter que les patients du groupe ayant obtenu des résultats équivoques à la PCR étaient moins susceptibles de se faire prescrire un macrolide (RC ajusté : 0,04; IC à $95 \%$ : 0,01 à 0,11 ), après ajustement en fonction de l'âge et de la présence de pneumonie à la radiographie. En fait, seulement $25 \%$ des cas équivoques ont été traités pour la coqueluche, même si la durée de leurs symptômes était comparable à celle observée chez les patients du groupe ayant obtenu des résultats positifs à la PCR. De plus, malgré une proportion similaire d'hospitalisations dans les deux groupes, les patients ayant obtenu des résultats équivoques à la PCR étaient hospitalisés moins longtemps (3,3 c. 12,2 jours, $p=0,001)$ et n'ont pas eu besoin d'être admis aux soins intensifs.

\section{Tableau 3 : Issues cliniques des patients}

\begin{tabular}{|c|c|c|c|c|c|}
\hline $\begin{array}{l}\text { Issues } \\
\text { cliniques }\end{array}$ & $\begin{array}{c}\text { Résultats } \\
\text { positifs à } \\
\text { la PCR } \\
n=109 \\
(\%)^{a}\end{array}$ & $\begin{array}{l}\text { Résultats } \\
\text { équivoques } \\
\text { à la PCR } \\
n=24(\%)^{a}\end{array}$ & $\begin{array}{c}\text { Valeur } \\
p\end{array}$ & $\begin{array}{c}\text { RC brut } \\
\text { (IC à } 95 \%)\end{array}$ & $\begin{array}{l}\text { RC ajusté } \\
\text { (IC à } 95 \%)\end{array}$ \\
\hline $\begin{array}{l}\text { Traitement par } \\
\text { l'amoxicilline }\end{array}$ & $0(0,0)$ & $4(16,7)$ & $<0,001^{\mathrm{b}}$ & S.O. & S.O. \\
\hline $\begin{array}{l}\text { Traitement par } \\
\text { un macrolide }\end{array}$ & $97(90,0)$ & $6(25,0)$ & $<0,001^{b}$ & $\begin{array}{r}0,04 \\
(0,01-0,12)^{\mathrm{a}}\end{array}$ & $\begin{array}{r}0,04 \\
(0,01-0,11)^{c}\end{array}$ \\
\hline Hospitalisation & $20(18,3)$ & $7(29,2)$ & 0,24 & $\begin{array}{r}1,81 \\
(0,63-4,83)\end{array}$ & $\begin{array}{r}4,63 \\
(0,75-47,96)^{d}\end{array}$ \\
\hline $\begin{array}{l}\text { Durée } \\
\text { moyenne du } \\
\text { séjour (É.-T.) }\end{array}$ & $12,2(10,2)$ & $3,3(1,0)$ & $0,001^{b}$ & S.O. & S.O. \\
\hline Séjour à I'USI & $7(6,4)$ & $0(0,0)$ & 0,20 & S.O. & S.O. \\
\hline Décès & $0(0,0)$ & $0(0,0)$ & S.O. & S.O. & S.O. \\
\hline $\begin{array}{l}\text { Deuxième } \\
\text { consultation }\end{array}$ & $21(19,3)$ & $4(16,7)$ & 0,73 & $\begin{array}{r}0,82 \\
(0,22-2,45)\end{array}$ & $\begin{array}{r}0,81 \\
(0,22-2,45)^{c}\end{array}$ \\
\hline
\end{tabular}

Abréviations : É.-T., écart-type; IC, intervalle de confiance; RC, rapport de cotes; S.O., sans objet; USI, unité de soins intensifs; $<$, inférieur à

a Á l'exception de la durée du séjour, qui est indiqué sous forme de moyenne avec écart-type

${ }^{b}$ Résultats statistiquement significatifs $(p<0,05)$

cAjusté en fonction de l'âge et de la présence de pneumonie à la radiographie

${ }^{d}$ Ajusté en fonction de l'âge

\section{Discussion}

Au CHUSJ, entre juin 2015 et mars 2017, les scores à la MPS et à l'indice RSS ont indiqué que les enfants ayant obtenu des résultats positifs ou équivoques au dépistage par PCR de $B$. pertussis présentaient certaines similitudes en ce qui concerne la gravité de la maladie. Toutefois, il existait de nombreuses différences importantes quant aux manifestations cliniques, aux résultats d'examens paracliniques et aux issues cliniques des patients entre les deux groupes. En fait, les patients du groupe ayant obtenu des résultats positifs à la PCR présentaient des symptômes caractéristiques de la coqueluche, comme l'apnée, la toux paroxystique, la toux émétisante et le chant du coq. La grande majorité des cas positifs ont été traités par un macrolide. Par contraste, les patients du groupe ayant obtenu des résultats équivoques présentaient plus souvent des symptômes non spécifiques d'infection des voies respiratoires supérieures, comme la rhinorrhée, la fièvre, un tirage et une respiration sifflante. De plus, la majorité des patients ayant obtenu des résultats équivoques n'ont pas été traités par un macrolide, ce qui laisse penser que le traitement contre la coqueluche n'était pas indiqué, selon le médecin traitant.

En utilisant la base de données des maladies à déclaration obligatoire de l'Ontario, Bolotin et ses collègues (5) avaient aussi déjà comparé les patients ayant obtenu des résultats positifs ou équivoques à la PCR. Ils avaient déclaré que les patients ayant obtenu un résultat équivoque à la PCR étaient moins susceptibles d'être hospitalisés que les patients ayant obtenu un résultat positif, même si les deux groupes présentaient un tableau clinique similaire. Dans le cadre de notre étude, les deux groupes se sont avérés significativement différents pour ce qui est des manifestations clinique et des issues, possiblement parce que notre population était composée d'enfants, lesquels sont souvent plus gravement touchés par la coqueluche que les adultes. DeVincenzo et ses collègues (9) ont également évalué le lien entre la valeur Ct à la PCR et la gravité de la coqueluche. Ils ont démontré que les valeurs $C t$ étaient significativement corrélées avec la durée de l'hospitalisation et la lymphocytose (9). Nos résultats suivent la même tendance.

D'un point de vue analytique, de nombreux facteurs peuvent expliquer les différences relevées entre les patients ayant obtenu un résultat équivoque et ceux ayant obtenu un résultat positif. La cible de la PCR pour le diagnostic de B. pertussis, I'IS481, est présente au nombre de 50 à 200 copies/cellule bactérienne. On a déjà démontré qu'une valeur Ct supérieur à 35 à la PCR pouvait représenter la détection de moins d'une bactérie par échantillon (10). La portée d'un résultat positif tardif demeure donc incertaine. D'une part, un résultat équivoque peut indiquer une véritable coqueluche avec une faible charge bactérienne, ce qui a pu être constaté dans le contexte d'une maladie durant plus de trois semaines, d'une vaccination antérieure, d'une immunité partielle ou de l'utilisation récente d'antibiotiques (2). Dans le cadre de notre étude, la durée des symptômes était semblable dans les deux groupes, ce qui rend peu probable I'hypothèse d'une maladie prolongée. Des échantillons de mauvaise qualité peuvent également produire des résultats équivoques. D'autre part, les résultats équivoques peuvent être causés par une colonisation transitoire où $B$. pertussis n'est pas lié au syndrome clinique. Waters et ses collègues ont décrit une éclosion de coqueluche atypique survenue à Toronto en 2005 et 2006. Parmi les 189 cas de coqueluche, définis par une valeur Ct à la PCR inférieure à 40 , seuls $42 \%$ correspondaient à la définition clinique de la coqueluche et jusqu'à un tiers des patients avaient un résultat positif à l'égard d'un autre agent pathogène. La valeur $\mathrm{Ct}$ moyenne pour ces cas était de 38,41, ce qui a évoqué l'idée que ces cas pouvaient représenter une colonisation transitoire (11). Par conséquent, Papenburg et Fontela ont postulé un lien entre les valeurs $\mathrm{C} t$ élevées et la présence de co-infections par des agents pathogènes respiratoires (12). Dans le cadre de notre étude, malgré le fait que la PCR multiplex virale ait été effectuée chez un nombre relativement faible de patients dans les deux groupes, les 
co-infections virales étaient quatre fois plus probables chez les patients ayant obtenu des résultats équivoques à la PCR.

Cette vaste étude rétrospective visait à évaluer des cas consécutifs d'enfants ayant fait l'objet d'une PCR multiplex bactérienne en raison de symptômes respiratoires sur une période de 22 mois dans un hôpital de soins tertiaires de Montréal. L'une des limites de notre étude était le recours à un examen manuel des dossiers pour la collecte de données, comprenant des notes manuscrites qui auraient pu être interprétées différemment par les chercheurs. Cependant, $10 \%$ des dossiers ont été examinés par deux membres de l'équipe et la concordance inter-évaluateurs était forte (coefficient kappa $=0,86)(13)$. Une autre limite réside dans le fait qu'il s'agit d'une étude uni-centrique; les patients qui ont fait l'objet d'investigations au CHUSJ ont pu aller en consultation dans n'importe quel autre établissement pour y obtenir un traitement dans les jours suivant leur visite - ces données seraient impossible à recueillir. Toutefois, notre série de cas décrit 12,5\% de tous les cas de coqueluche signalés au Québec pendant la période de l'étude (14).

\section{Conclusion}

Malgré des similitudes quant à la gravité de la maladie, les manifestations cliniques chez les enfants avec suspicion clinique de coqueluche et ayant obtenu des résultats équivoques à la PCR était significativement différent de celui observé chez les enfants ayant obtenu des résultats positifs. Dans un contexte où les ressources de santé publique sont limitées, ce constat peut orienter la décision concernant la nécessité de signaler ou non les résultats équivoques aux autorités de la santé publique.

\section{Déclaration des auteurs}

M. D. - conceptualisation, méthodologie, validation, recherche, conservation des données, rédaction - ébauche originale, examen et révision de la version définitive

S. M. - conceptualisation, méthodologie, validation, recherche, examen et révision de la version définitive

P. D.-P. - analyse officielle, examen et révision de la version finale N. B. - méthodologie, examen et révision de la version finale D. I. - recherche, conservation des données, examen et révision de la version finale

F. R. - recherche, ressources, examen et révision de la version finale

C. Q. - conceptualisation, méthodologie, examen et révision de la version finale, supervision, acquisition de financement

\section{Conflit d'intérêts}

Aucun

\section{Financement}

La Dre Quach a reçu un soutien salarial externe (FRQ-S mérite, subvention $\left.n^{\circ} 252775\right)$.

Ces travaux de recherche ont été rendus possibles par le Centre de recherche du CHU Sainte-Justine (fonds de démarrage de c. Quach).

\section{Références}

1. Douville-Fradet M, Amini R. Épidémiologie descriptive des maladies à déclaration obligatoire évitables par la vaccination au Québec, 2011-2013. Institut National de Santé Publique du Québec. 2016. www.inspq.qc.ca/sites/default/ files/publications/2198_maladies_evitables_vaccination_ declaration_obligatoire.pdf

2. Brousseau N, Judd L, Marcoux-Huard C, Landry M. Guide d'intevention - La coqueluche. Ministère de la santé et des services sociaux du Québec. 2017. http://publications.msss. gouv.qc.ca/msss/fichiers/2017/17-271-03W.pdf

3. Ebell MH, Marchello C, Callahan M. Clinical Diagnosis of Bordetella Pertussis Infection: A Systematic Review. J Am Board Fam Med 2017 May-Jun;30(3):308-19. https://doi. org/10.3122/jabfm.2017.03.160330. PubMed (https://www. ncbi.nlm.nih.gov/pubmed/28484063?dopt=Abstract)

4. Mattoo S, Cherry JD. Molecular pathogenesis, epidemiology, and clinical manifestations of respiratory infections due to Bordetella pertussis and other Bordetella subspecies. Clin Microbiol Rev 2005 Apr;18(2):326-82. https://doi.org/10.1128/ CMR.18.2.326-382.2005. PubMed (https://www.ncbi.nlm.nih. gov/pubmed/15831828?dopt=Abstract)

5. Bolotin S, Deeks SL, Marchand-Austin A, Rilkoff H, Dang V, Walton R, Hashim A, Farrell D, Crowcroft NS. Correlation of Real Time PCR Cycle Threshold Cut-Off with Bordetella pertussis Clinical Severity. PLoS One 2015 Jul;10(7):e0133209. https://doi.org/10.1371/journal. pone.0133209. PubMed (https://www.ncbi.nlm.nih.gov/ pubmed/26186564?dopt=Abstract)

6. Public Health Ontario. Bordetella testing by Real-Time PCR 2012. Toronto ON; Public Health Ontario: April 2012. www.publichealthontario.ca/en/eRepository/LAB_SD_078 Bordetella_testing_real_time_PCR.pdf

7. Gill CJ, Mwananyanda L, MacLeod W, Kwenda G, Mwale M, Williams AL, Siazeeke K, Yang Z, Mwansa J, Thea DM. Incidence of Severe and Nonsevere Pertussis Among HIV-Exposed and -Unexposed Zambian Infants Through 14 Weeks of Age: Results From the Southern Africa Mother Infant Pertussis Study (SAMIPS), a Longitudinal Birth Cohort Study. Clin Infect Dis 2016 Dec;63(suppl 4):S154-64. https://doi. org/10.1093/cid/ciw526. PubMed (https://www.ncbi.nlm.nih. gov/pubmed/27838668?dopt=Abstract)

8. Feldman AS, Hartert TV, Gebretsadik T, Carroll KN, Minton PA, Woodward KB, Larkin EK, Miller EK, Valet RS. Respiratory Severity Score Separates Upper Versus Lower Respiratory Tract Infections and Predicts Measures of Disease Severity. Pediatr Allergy Immunol Pulmonol 2015 Jun;28(2):117-20. https://doi. org/10.1089/ped.2014.0463. PubMed (https://www.ncbi.nlm. nih.gov/pubmed/26155369?dopt=Abstract)

9. DeVincenzo JP, Guyton C, Rea H, Elmore E, Patel S, Wynn L, Harrison L, El Saleeby CM, Bagga B. Molecular detection and quantification of pertussis and correlation with clinical outcomes in children. Diagn Microbiol Infect Dis 2013 May;76(1):10-5. https://doi.org/10.1016/j. diagmicrobio.2012.12.015. PubMed (https://www.ncbi.nlm.nih. gov/pubmed/23490009?dopt=Abstract)

10. Guthrie JL, Seah C, Brown S, Tang P, Jamieson F, Drews SJ. Use of Bordetella pertussis BP3385 to establish a cutoff value for an IS481-targeted real-time PCR assay. J Clin Microbiol 2008 Nov;46(11):3798-9. https://doi.org/10.1128/ 
JCM.01551-08. PubMed (https://www.ncbi.nlm.nih.gov/ pubmed/18784312?dopt=Abstract)

11. Waters $V$, Jamieson $F$, Richardson SE, Finkelstein $M$, Wormsbecker A, Halperin SA. Outbreak of atypical pertussis detected by polymerase chain reaction in immunized preschool-aged children. Pediatr Infect Dis J 2009 Jul;28(7):582-7. https://doi.org/10.1097/ INF.0b013e318197fac1. PubMed (https://www.ncbi.nlm.nih. gov/pubmed/19561423?dopt=Abstract)

12. Papenburg J, Fontela P. What is the significance of a high cycle threshold positive IS481 PCR for Bordetella pertussis? Pediatr Infect Dis J 2009 Dec;28(12):1143. https://doi.org/10.1097/
INF.0b013e3181bd4e1f. PubMed (https://www.ncbi.nlm.nih. gov/pubmed/19935278?dopt=Abstract)

13. McHugh ML. Interrater reliability: the kappa statistic. Biochem Med (Zagreb) 2012;22(3):276-82. https://doi.org/10.11613/ BM.2012.031. PubMed (https://www.ncbi.nlm.nih.gov/ pubmed/23092060?dopt=Abstract)

14. Ministère de la Santé et des Services sociaux. Vigie et surveillance des maladies à déclaration obligatoire d'origine infectieuse. Rapport annuel 2016. MSSS: 2017. http:// publications.msss.gouv.qc.ca/msss/fichiers/2017/17-268-05W. pdf

\section{DES MILLIERS DE VOLONTAIRES NOUS

Inscrivez-vous au programme « La grippe

à l'œil » dès aujourd'hui et faites parties de la nouvelle tendance en surveillance de la grippe.
Vous pouvez fournir de l'information utile qui servira à améliorer la santé publique dans votre communauté et partout au pays.
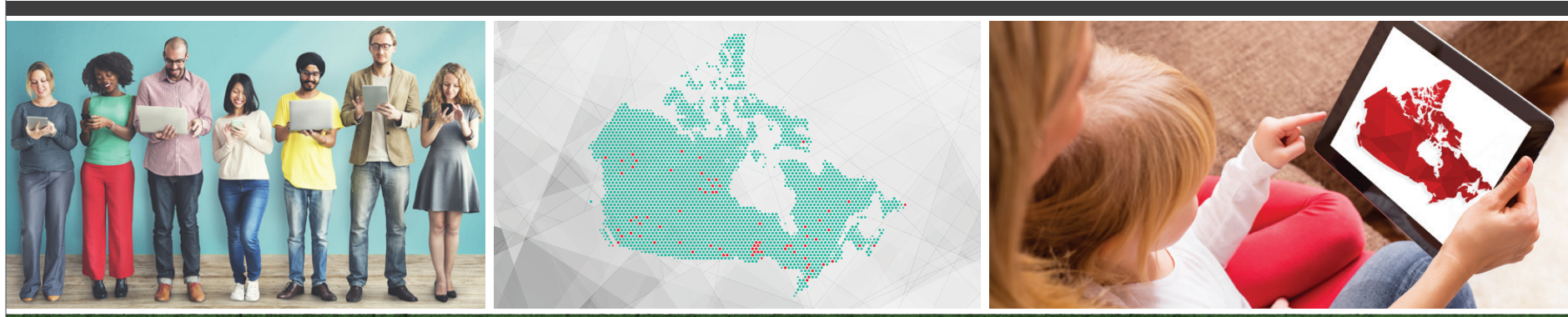

POUR EN APPRENDRE DAVANTAGE AU SUJET DU PROGRAMME «LA GRIPPE À L' EIL », VISITEZ LE SITE WEB CANADA.CA/GRIPPE. 